1

$19^{\text {TH }}$ WPA WORLD CONGRESS OF PSYCHIATRY LISBON, 21-24 AUGUST, 2019

\section{Combined cognitive and neuromodulation intervention in physiological and pathological aging: effects on frontal executive functions}

\author{
Davide Crivelli1,2, Monica Zanca ${ }^{3}$, Michela Balconi ${ }^{1,2}$
}

1 Department of Psychology, Catholic University of Milan, Milan, Italy 2 Research Unit in Affective and Social Neuroscience, Catholic University of Milan, Milan, Italy 3 Fondazione "le Rondini" Città di Lumezzane Onlus, Brescia, Italy

\title{
Introduction
}

Aging, besides being characterized by increased psycho-physical frailty, is now considered a dynamic process showing potential for adaptive modifications of neural and cognitive processes. Such compensatory mechanisms might be fostered by appropriate neurocognitive interventions, via both cognitive training and non-invasive brain stimulation to promote neural plasticity.

The opportunities offered by the integration of different techniques are, however, still understudied. Therefore, we devised and tested an integrated neurocognitive enhancement protocol in both physiological and pathological aging.

Method

AIM $\rightarrow$ The present work aimed at investigating the potential for optimizing cognitive skills in aging of an integrated neurocognitive enhancement protocol, which combined prefrontal transcranial electrical stimulation and computerized cognitive training targeting higher executive functions.

SAMPLE $\rightarrow 21$ healthy elderly and 22 patients presenting clinical signs of Alzheimer's Disease, divided into an experimental group, which trained with computerized exercises while being administered bilateral prefrontal neuromodulation, and a control group, which only trained with computerized exercises and received sham stimulation

\begin{tabular}{|cccccc|}
\hline & $\mathbf{N}$ & Mage & SDage & Edu & SDEdu \\
\hline Patients & 22 & 84,68 & 6,59 & 4,56 & 1,41 \\
\hline Healthy Elderly & 21 & 66,77 & 5,85 & 8,77 & 3,84 \\
\hline Total sample & $\mathbf{4 3}$ & $\mathbf{7 5 , 7 3}$ & $\mathbf{1 0 , 9 1}$ & $\mathbf{7 , 0 0}$ & 3,70 \\
\hline
\end{tabular}

\section{Analysis of pre-/post-training data highlighted:}

1. different profiles of cognitive improvement between patients and healthy controls

2. combining cognitive training with prefrontal neuromodulation seemed to strengthen such pattern of observed outcomes

3. Increased or better-preserved markers of EEG reactivity in the experimental group, suggesting a specific effect on the sensitivity to environmental information
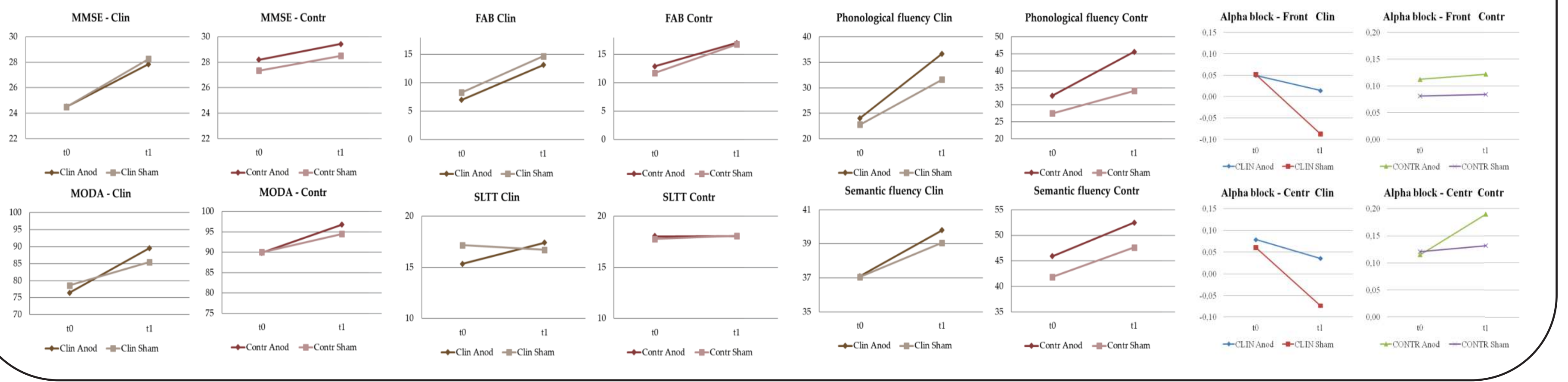

\section{Discussion}

In addition to an improvement of cognitive functions, there were interesting qualitative changes in relationships and behavioral correlates.

Comparing the general trends of the group of patients and of the group of healthy elderly, it is possible to notice that although both groups present profiles of global improvement and dependent on specific cognitive functions, the clinical sample seems to benefit a little more than integrated training if we consider some aspects of the frontal executive functions.

This is consistent with the functional DLPFC role in aging. Moreover, future studies might investigate if these findings are related to the neuro-enhancement of metacognitive executive control functions.

NB1 $\rightarrow$ Possible advantage of the integration with neuromodulation when working with people in pathological aging - cfr. role of margin of improvement?

NB2 $\rightarrow$ On the whole, intensive neurocognitive empowerment protocols might help improving cognitive skills in elderly people in line with the role of prefrontal cortex in supporting executive control

\section{References}

Antonietti, A. Balconi. M., Catellani, P. \& Marchetti, A. (2014). Empowering Skills for an Active Ageing and Healthy Living In G. Riva, Cabeza. R. (2002). Hemispheric asymmetry reduction in older adults: the HAROLD model. Psychology and Aging, 17(1) 85-100. P. Ajmone Marsan, \& C. Grassi (Eds.), Active Aging and Healthy Living (pp. 157-171). Amsterdam: IOS Press. 